the dissection is carried out with a blunt instrument kept close to the gland substance. The gland is usually tightly adherent to the trachea at one point in front, and though a blunt instrument has been used to separate it at the sides, the scissors will nearly always be necessary to effect the separation from the front and central portion of the trachea. There is no need to lay stress on the special difficulties of this operation-the true exophthalmic gland has outlying lobes and nodules, and is very hard, exceedingly vascular, friable, and adherent. This may render very difficult the process of dislocating the lobe and lifting it up to separate it from the trachea, while the patients, if the disease is at all advanced, stand loss of blood very badly, and every effort must be made to diminish haemorrhage. The veins are usually more troublesome than the arteries-as in all branches of surgery-and though a local anaesthetic has the effect of making the superficial tissues avascular, it has no such effect on the deeper ones. Further, the fact that the patient is not unconscious makes a haemorrhage, should it occur, more difficult to deal with.

The mortality of this operation in the last 900 cases has been $2 \frac{1}{2}$ per cent.

\section{Results of Treatment}

It may well be asked, "What are we to expect from this operation in the way of improvement? "and the first question that the patient will ask is probably about the mortality. A mortality of $2 \frac{1}{2}$ per cent. is not unduly high when one is dealing with so serious a complaint. This disease leads inevitably to prolonged and often very serious ill-health, and gives rise to symptoms which are most unpleasant to the patient and even to her friendsespecially the incessant palpitation and rapid action of the heart, the nervousness, jumpiness, and the sleeplessness. Moreover, in many cases-especially in women over 40-it leads to gross disorganization of the heart and to death. It is without doubt justifiable to run a small amount of risk in an attempt to effect a cure. Nevertheless, one cannot help regarding all patients with a certain amount of anxiety for the first two or three days after operation.

Of the twenty-two patients in this series who succumbed eight died of pneumonia within the first four days, and the other fourteen within sixty hours of the operation with the symproms of what one is accustomed to call acute hyperthyroidism. After three or four days, therefore, any danger of death would seem to be over. Following the operation there usually appears to be an improvement, which is more marked and rapid in the subjective symptoms than in the physical signs, and patients often look and feel much better within a few days of the thyroidectomy. It is often not for a month or so that really marked improvement in the physical signs sets in. This improvement in subjective symptoms often disappears after a few weeks, when the patient goes back to her own home, but reappears later as a permanency in two or three months, and is then accompanied by marked improvement in the physical signs. A long rest for some months after the operation is absolutely essential.

As a rule, after three or four months we find that patients who have had an adequate amount of thyroid gland tissue removed feel well, eat well, sleep well, and can do their day's work, though I do not think it wise for them to recommence their normal life quite as soon as this. Though thè look and feel well and put on weight, it is frequently found on examination that the pulse rate, while very much slower than it used to be, has not quite come down to the normal, remaining between 80 and 90 , and that the cardiac dilatation, though considerably less, has not quite gone. Irregularity of the pulse, acetonuria, and diarrhoea, if previously present, almost invariably disappear, and the nervous excitability, tremor, and jumpiness are greatly improved; but the exophthalmos, though much less marked than before operation, rarely if ever vanishes completely. Those patients who receive surgical treatment for the condition comparatively early in its course-that is, before they have developed the gross degenerative conditions of the myocardium and of other viscera that set in sooner or later-should all, if a sufficient amount of thyroid tissue is removed, become well in the sense that they should be able to lead a normal life, do their ordinary work, and sleep the sleep of the just. On the other hand, it is no use pretending that operation on a case with a grossly dilated and fibrillating heart and advanced myocardial degeneration will ever make that heart perfect again, although it will make it very much better. Palpitation and shortness of breathtwo symptoms especially unpleasant to the patientusually show the greatest amount of improvement.

As regards the effect of the operation on the loss of weight, which is often so serious a feature in a bad case, the gain in weight following operation is nearly always marked, and may even become embarrassing. Another very satisfactory feature about this branch of surgery is that, as a general rule, the bad cases appear to derive more improvement from, and to be even more pleased with the results of, the operation than do the slighter cases.

As in other diseases, it is difficult to compare and contrast the results of treatment by merely observing symptoms and physital signs, and it is therefore satisfactory to find that there appears to be a method by which one can attempt to measure what we may call the "physiological fitness" of these paitients before and after operation. Many of these patients have had their basal metabolic rates estimated before and after operation; before operation they are nearly always 40 to 80 per cent. above that of the normal individual-this presumably representing increased tissue metabolism as a result of the hyperactivity of the thyroid. After the operation these rates fall, within a few weeks, to amounts varying from normal to +25 per cent. above normal, and this appears to be a fixed method of estimating the " physiological improvement." There have so far been only two or three cases in which, after operation, this basal metabolic rate has fallen slightly below normal, to amounts varying between -5 and -10 per cent., and these patients have not, up to the present, shown any signs clinically of hypothyroidism.

Of the success of surgery in suitable cases of Graves's disease there is, I think, no doubt, but one cannot help feeling conscious that in removing thyroid tissue one is really treating symptoms and not causes, and that the primary cause is something further back and not yet removable.

\section{THREE RECENT CASES OF ENCEPHALITIS LETHARGICA}

BY

PHILIP FRAZER, M.B.

HOUSE-PHYSICIAN, GENERAL HOSPITAL, BIRMINGHAM

\section{Introductory Note by}

A. STANLEY BARNES, M.D., F.R.C.P. Senior Physician, General Hospital, Birmingham

During the last three years a comparatively small number of cases of encephalitis lethargica have been notified. Considerable doubt has existed in the minds of many as to how far these notified cases are really encephalitis, seeing that the difficulty of diagnosis is unusual in this disease. In the last few months there have been admitted under my care at the General Hospital, Birmingham, three cases of a somewhat fulminating character in which a post-mortem examination has permitted confirmation of the diagnosis. The type of disease in these cases resembles to some extent that which was seen in the very early stages of the epidemic in 1918. Very few of such cases have been seen in the Midlands during the last few years. It is of interest to note the existence of this severe type of case during this year, as it is possible this means that a further visitation of encephalitis in epidemic form 
may be imminent; it is equally possible that these are just stray cases which indicate that the epidemic has spent its force, and that in future we shall see only occasional cases appearing in sporadic form.

\section{CASE I}

A road foreman, a previously healthy man, aged 57, was admitted to the General Hospital, Birmingham, on May 30th, 1932, complaining of headache, giddiness, and double vision, with some numbness of the hands, for fourteen days. The illness began while he was at work, with sudden inability to see more than a few yards, and on reaching home he found he was seeing double. After two days' rest in bed the double vision disappeared. On admission he complained of a dull, heavy sensation in his head, and a feeling of giddiness and insecurity when on his feet. He had had some nausea, but no vomiting. Sleep was good.

Examination.-The patient was found to be a well-nourished man; he gave an intelligent history, but his speech was slow and indistinct. Bilateral ptosis and right facial weakness were apparent as he lay in bed. The pupils reacted to light, but not to accommodation. Both fundi were normal. Both internal rectus oculi muscles were paralysed, and irregular nystagmus was present on looking upwards and downwards. There was slight weakness of the muscles of mastication on the right side and a definite right facial weakness. The other cranial nerves were unaffected. There were no other motor or sensory changes present. The other systems were normal. Lumbar puncture three days after admission revealed a clear, colourless fluid, not under pressure: cells, less than 1.5 per c.mm.; sugar, $68 \mathrm{mg}$. per $100 \mathrm{c} . \mathrm{cm}$. ; protein, $85 \mathrm{mg}$. per $100 \mathrm{c.cm}$. (globulin negative) ; chlorides, $730 \mathrm{mg}$. per $100 \mathrm{c.cm}$. ; Lange curve, 1233210000 ; Wassermann reaction, negative.

Progress.-On June 8th the patient became irrational, and from this time drowsiness and lethargy became a prominent feature of the disease. On June 10th the patient was able to swallow only with difficulty, and articulation was very indistinct. By this time a complete ophthalmoplegia had developed-the eyes looking straight forward-but the pupils still reacted to light. The drowsiness had now become constant. Shortly after this, paralysis of the muscles of deglutition supervened, and nasal feeding was resorted to. On June 16 th the patient was comatose, with Cheyne-Stokes respiration. All four limbs were slightly rigid, the abdominal reflexes were absent, the tendon-jerks exaggerated, and both plantar responses extensor. There was incontinence of urine and facces. Though previously afebrile he now began to run a temperature varying between $101^{\circ}$ and $102^{\circ}$. He remained in this condition until he died, four days later.

Post-mortem.-The brain surface was moist, the dura mater was not adherent, and there was no flattening of the convolutions. A horizontal section across the cerebral hemispheres revealed no macroscopical abnormality, but the brain stem and basal ganglia showed muitiple small haemorrhagic spots, which were particularly obvious in the grey matter surrounding the Sylvian aqueduct and in the floor of the fourth ventricle. The appearances were typical of encephalitis lethargica. The lungs showed an early hypostatic pneumonia; the other organs were normal. Microscopical sections taken from the region of the basal ganglia, the mid-brain, pons, and medulla also showed the appearances expected in this condition-namely, large collections of lymphocytes in the perivascular spaces of the smaller vessels. There was also degeneration of the ganglion cells in several areas.

\section{$\dot{\mathrm{C}}_{A S E}$ II}

A married woman, aged 52, was admitted to hospital on August 8th, 1932, after a fortnight's illness. The initial symptoms were of feeling tired and weak, with rapid pulse and an occasional rise in temperature. A few days before admission she became delirious. No physical signs were discovered at any time by her doctor.

Examination and Progress.-The patient was delirious and very restless; temperature $99^{\circ}$, pulse 110 , respirations 27. A complete neurological examination was out of the question owing to the extreme restlessness and irritability of the patient, but as far as could be made out there was no paralysis of the cranial nerves, trunk, or limbs. The other systems were normal. Lumbar puncture was performed under light ether anaesthesia. The fluid obtained was clear, colourless, under a tension of $235 \mathrm{~mm}$. of water, and contained no organisms. The cell content was less than 1 per c.mm.; sugar, $57 \mathrm{mg}$. per $100 \mathrm{c.cm}$.; protein, $70 \mathrm{mg}$. per $100 \mathrm{c.cm}$. ; chlorides, $745 \mathrm{mg}$. per $100 \mathrm{c} . \mathrm{cm}$.; urea $111 \mathrm{mg}$. per 100 c.cm.; Wassermann reaction, negative. There was no return of consciousness following the anaesthetic, and the patient died ten hours later with pin-point pupils, a temperature of $107^{\circ}$, pulse of 160 , and respiration of 60 .

Post-mortem.-The whole of the grey matter exhibited a slightly pink colour; this was particularly noticeable in the tissues of the basal ganglia and in those of the brain stem, where the brain substance was also unusually soft. The grey matter around the Sylvian aqueduct and the floor of the fourth ventricle showed minute haemorrhagic spots, and larger haemorrhages were to be seen in the fillet and elsewhere in the brain stem. Sections taken from the mid-brain, pons, and medulla for microscopy showed local areas of oedema and softening of the brain substance with vascular congestion. No perivascular " cuffing" by lymphocytic infiltration was seen. Considerable neuronic degeneration was present.

\section{CASE III}

A married woman, aged 39, was admitted to hospital on September 5th, 1932, after one week's illness, consisting of headache, loss of appetite, and constipation. Two days before admission she became delirious, and then completely negative mentally.

Examination and Progress.-The patient was a well-nourished woman; she was lying very still in bed and occasionally moving her head from one side to the other. There was rhythmic blinking of the eyelids, which persisted the whole time until the patient died. The jaws were tightly clenched and swallowing movements were entirely absent, the saliva dribbling from the patient's mouth. A certain amount of head retraction was present, and the arms were flexed at the elbows and rigid. The patient would not obey any command, and did not react to painful stimuli. Indeed, lumbar puncture performed without a local anaesthetic evoked only a slight flinching movement. The temperature was $103^{\circ}$, pulse 90 , and respirations 25. Both fundi oculi were normal; there was no ophthałmoplegia or facial weakness. There was a certain amount of rigidity present in the arms-chiefly in the flexor muscles-which diminished on manipulation. The arm-jerks were brisk; the abdominal reflexes, both superficial and deep, were absent; the legs were slightly rigid with diminished reflexes, and the plantar responses were flexor. There was complete incontinence of urine and faeces. The other systems were normal. Lumbar puncture was performed on the following day, and 6 c.cm. of faintly blood-stained fluid were removed. The analysis was as follows: cells, mostly polymorphs, 70 per c.mm., a little blood present; no T.B. or other organisms seen. The supernatant fluid of the centrifugalized specimen contained: sugar, $96 \mathrm{mg}$. per $100 \mathrm{c.cm}$.; protein, $170 \mathrm{mg}$. per $100 \mathrm{c} . \mathrm{cm}$. (globulin positive); chlorides, $722 \mathrm{mg}$. per 100 c.cm. The patient's mental condition remained the same throughout; she uttered no sound, but made snarling and twitching movements with her lips. Rhythmic movements of the eyes persisted, and dissociated movements of the eyes themselves soon appeared. Nasal feeding was resorted to as the patient was unable or refused to swallow. The patient died on September 8th-that is, three days after admission-the temperature rising to $108^{\circ}$.

Post-mortem.-On removing the skull vault and dura mater the brain convolutions showed extreme congestion without any flattening, oedema, or exudate. Examination of the cerebral cortex, basal ganglia, mid-brain, pons, and medulla revealed great congestion, the grey matter showing a distinct pink coloration. In the anterior part of the corpus callosum, close to the anterior pillars of the fornix, there was a welldefined small haemorrhage; this was more than a petechial haemorrhage. Throughout the grey matter of the brain stem there were innumerable tiny points of haemorrhage ; they were particularly well marked in the grey matter arovnd the Sylvian aqueduct and in the floor of the fourth vertricle.

In the heart there was an area of recent haemorrhage under the endocardium of the septal wall of the left ventricle and many subpericardial petechiae. The lungs were very congested; both the lower lobes were partially consolidated by very definite areas of bronchopneumonia. There were alsc 
many small subpleural haemorrhages. The other organs were normal. Microscopically the appearances were characteristic of encephalitis lethargica. The sections of the mid-brain, pons, and medulla showed the following features: great congestion of all the vessels with infiltration of the perivascular spaces, which were also dilated. This perivascular cuffing was most marked in the sections from the pons and medulla. Haemorrhages were seen in various parts, a very large one being present near the lamina terminalis. The ganglion cells in the brain stem showed all degrees of degeneration-some of it pigmentary-and loss of nuclear staining.

\section{COMMENTARY}

Three cases of encephalitis lethargica, proved at postmortem, are described. In all of them there was a prodromal period lasting from one to two weeks, consisting of malaise, headache, lethargy, and constipation.

The first case pursued the characteristic course of a subacute disease, with its maximal incidence on the midbrain, spreading thence upwards to the hypothalamic region and basal ganglia, and downwards to the pons and medulla oblongata.

Cases II and III were similar to one another in type. Both were remarkable for the fulminating course of the disease once the prodromal period was passed, the acute stage lasting only four days or so. Both showed a complete mental obliteration, and both terminated similarly with a hyperpyrexia.
The macroscopic post-mortem findings were typical of encephalitis lethargica, the main points being the pink discoloration of the grey matter, the vascular congestion, and the presence of multiple minute haemorrhages, giving the brain a flea-bitten appearance. This was especially noticeable in the region of the basal ganglia and in the brain stem. The exceptional severity of the disease in Case III was attested by the presence of haemorrhages beneath the endocardium, pericardium, and pleura-a finding to be expected where an acute toxic process has been at work.

Microscopically the characteristic cuff of lymphocytic infiltration in the perivascular spaces was present in two of the cases. At the same time there was degeneration, often pigmentary in nature, of the nerve cells. The sections also showed a certain amount of spreading apart of the neuroglial elements due to oedema. Although the sections of Case II did not show the lymphocytic cuffing of the small vessels, neuronic degeneration was a marked feature, and the similarity of the clinical course of the disease to that of Case III, taken in conjunction with the macroscopic appearance of the brain, leaves little room for doubt as to the true nature of the condition.

I wish to thank Dr. Stanley Barnes and Dr. A. Brian Taylor for their assistance and advice in the writing of this paper, and Dr. F. W. Mason Lamb, pathologist to the General Hospital, Birmingham, who performed the necropsies and reported on the histological sections.

\section{PAIN REFERRED TO THE EAR* BY}

E. WATSON-WILLIAMS, M.C., CH.M., F.R.C.S.Ed. SURGEON IN CHARGE, EAR, NOSE, AND THROAT DEPARTMENT, BRISTOL ROYAL INFIRMARY ; LARYNGOLOGIST, BRISTOL AND SOUTH-WESTERN RADIUM CENTRE

Pain felt in an otherwise apparently normal ear presents a diagnostic problem often of some interest. I say " apparently normal " since at times the membrane shows no appreciable change, even when mastoid suppuration or yet more serious trouble is present. ${ }^{5}$ But when the earache is really due to disease elsewhere, if on examining the ear we find some lesion possibly sufficient to account for the pain it is only too easy to walk into the trap-particularly where the pain is severe or the patient manifestly ill and we feel compelled to adopt active measures without delay. The pain may be due to trouble near but not in the ear, such as arthritis of the temporomandibular joint. Earache is not infrequently the first symptom of mumps, and may occur before any swelling is noticed. Even a mixed tumour of the parotid may have earache as the only symptom.

\section{Herpes Oticus}

Herpes oticus provides another possible catch. The pain is intense, sometimes with great tenderness of the meatus and mastoid, and may precede the eruption by several days-among ten cases I found intervals of six, four, and three days. There is some fever ; in one case the temperature was $102^{\circ} \mathrm{F}$. Deafness, nausea, and slight vertigo are common, and the membrane may be a trifle pink. Points of diagnostic importance are a constant boring ache-as contrasted with the fluctuating, throbbing pain of otitis-and extreme tenderness of the skin to very light touch. Even when a vesicle simulates a perforation, careful examination will reveal the difference, the discharge being slight and sanious; sometimes a history of exposure to varicella is obtained. No doubt the majority of cases of herpes oticus are due to involvement of the geniculate ganglion, ${ }^{2}$ though in my experience only one-third have facial paresis, and I have only once

* Opening paper to discussion on referred pain, at a meeting of the Sections of Otology and Laryngology of the Royal Society of Medicine, held at Portsmouth, June, 1932. seen vesicles on the tongue. Twice I have seen an eruption on the soft palate, with dysphagia, which is perhaps evidence of glosso-pharyngeal affection. Herpes oticus with paralysis of the vocal cord has been recorded. ${ }^{3}$. The two following cases illustrate vagus herpetic earache, and the third case vagus referred earache without herpes.

CASE I.-Earache from Herpes Oticus, with Pneumonia

W. H., aged 14, had severe pain in the left ear for three days, following a cold. He had become much worse, with giddiness, vomiting, and a temperature of $104.4^{\circ} \mathrm{F}$. and pulse of 120. There was slight sanious oral discharge and extreme tenderness of the mastoid tip. I was sent for late at night to perform an operation. When I saw the boy in the anaesthetic room I was struck by the "pneumonia" lookflushed cheeks, hot dry skin, bright eye, etc. The ear showed what looked like a perforation just in front of the umbo, but on careful inspection I thought it was a ruptured vesicle. All that could be found in the chest were a few crepitations in the right base, but thirty-six hours later (not before) there were typical signs of lobar pneumonia.

CASE II.-Earache from Herpes Oticus, with Pneumonia

J. E. T., a man aged 31, had symptoms of fever, deafness, and right earache, worse on coughing, following influenza. He had typical pneumonia of the right upper lobe, and a herpetic eruption on the right tympanic membrane.

\section{CASE III.-Earache due to Pneumonia}

A boy of 10 came to the casualty department of the Royal Infirmary complaining of bad earache of a few hours' duration, following a "chill." There was no tenderness, and the ear appeared quite normal ; but the boy was obviously ill, and examination of the chest showed consolidation of the left lower lobe.

\section{Other Causes}

Neuritis is a not uncommon cause of earache. Exposure to cold is the usual aetiological factor, and there is sometimes facial paralysis in addition. I have seen a number of cases in taxi and lorry drivers, generally on the right side-probably because that ear is more exposed to the wind than the other. The trouble is usually transient, but some cases have proved very persistent. The pain of neuralgia is seldom confined to the ear ; indeed, it tends as a rule to be felt round rather than in it. True geniculate tic douloureux seems to be very rare; the pain is paroxysmal, confined to the ear, severe and lancinating in character. ${ }^{1}$ 